\title{
The Effect of Resveratrol on Blood Glucose and Blood Lipids in Rats with Gestational Diabetes Mellitus
}

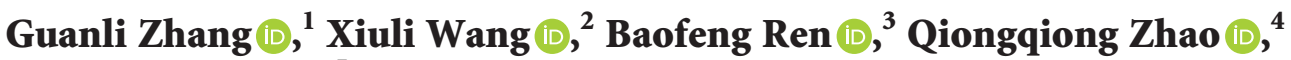 \\ and Fang Zhang (iD ${ }^{5}$ \\ ${ }^{1}$ Department of Obstetrics, Yantaishan Hospital, Yantai 264000, Shandong Province, China \\ ${ }^{2}$ Department of Obstetrics, Chengyang People's Hospital, Qingdao 266000, Shandong Province, China \\ ${ }^{3}$ Medical Insurance Department, Zhangqiu District People's Hospital, Jinan,250200, Shandong Province, China \\ ${ }^{4}$ Department of Paediatrics (II), Zhangqiu District People's Hospital, Jinan 250200, Shandong Province, China \\ ${ }^{5}$ Department of Clinical Laboratory, Jinan Central Hospital, Cheeloo College of Medicine, Shandong University, Jinan 250013, \\ Shandong Province, China
}

Correspondence should be addressed to Fang Zhang; luaofangx@163.com

Received 1 September 2021; Revised 9 October 2021; Accepted 11 October 2021; Published 21 October 2021

Academic Editor: Muhammad Wasim Khan

Copyright ( 92021 Guanli Zhang et al. This is an open access article distributed under the Creative Commons Attribution License, which permits unrestricted use, distribution, and reproduction in any medium, provided the original work is properly cited.

Background. Previous studies have reported that resveratrol has various biological effects such as anti-inflammatory, antioxidant, and antitumor. This study aimed to investigate the effects of resveratrol on blood glucose and blood lipids in rats with gestational diabetes mellitus (GDM). Methods. The rat diabetes model was prepared by one-time intraperitoneal injection of streptozotocin (STZ, $35 \mathrm{mg} /$ $\mathrm{kg}$ ). Fasting blood glucose was measured by using a blood glucose meter. The ELISA method was used to detect the levels of insulin, leptin, adiponectin, resistin, TNF- $\alpha$, and IL-6. The content of TC, TG, LDL-C, and HDL-C was determined by using an automatic biochemical detector. Results. Compared with the GDM group, the insulin level in the resveratrol (120 and $240 \mathrm{mg} / \mathrm{kg})$ treatment group was significantly increased. But, the blood glucose level and body weight were significantly reduced. The content of TC, TG, and LDL-C in the resveratrol $(240 \mathrm{mg} / \mathrm{kg})$ treatment group was significantly reduced, and the content of HDL-C was significantly increased. In addition, leptin, resistin, TNF- $\alpha$, and IL-6 levels in the $240 \mathrm{mg} / \mathrm{kg}$ resveratrol treatment group were significantly reduced, and adiponectin was significantly increased. Also, resveratrol $(240 \mathrm{mg} / \mathrm{kg})$ was stronger than metformin hydrochloride in improving insulin secretion and regulating blood lipids and adipokine content. Conclusion. Resveratrol has a dose-dependent effect on GDM rats to increase insulin secretion, reduce blood glucose and body weight, and regulate blood lipids and plasma adipokines.

\section{Introduction}

Gestational diabetes mellitus (GDM) refers to diabetes or impaired glucose tolerance that occurs or is first discovered during pregnancy [1]. Although the abnormal glucose metabolism of GDM patients can return to normal after delivery, the chance of developing type 2 diabetes will increase in the future [2]. GDM is extremely harmful to mothers and babies and may cause preeclampsia, premature rupture of membranes, and premature delivery [3]. The incidence of fetal malformations is 3 to 4 times that of the normal control group [4]. Therefore, timely treatment of
GDM is of great significance to the health of mothers and infants and can also effectively reduce the incidence of diabetes in the future.

The etiology and pathogenesis of GDM are extremely complex and have not yet been fully elucidated. The main features of glucose metabolism during pregnancy are increased glucose demand, increased insulin resistance, and relatively insufficient insulin secretion [5]. This may cause GDM in some pregnant women. GDM is more common in people who are obese or overweight, people who have a longterm high-sugar and high-fat diet, people who have a family history of diabetes, and elderly pregnant women [6]. 
Hyperglycemia can cause abnormal embryonic development and even death, and the incidence of miscarriage can reach $15 \%$ to $30 \%$ [7]. GDM patients are 2 to 4 times more likely to develop high blood pressure during pregnancy than nondiabetic pregnant women. This may be related to the presence of severe insulin resistance and hyperinsulinemia [8]. In addition, it has been found that obesity, lipid metabolism disorders, and abnormal secretion of adipocytokines in adipose tissue play a very critical role in the pathogenesis of GDM [9]. Therefore, it is very important for GDM patients to find drugs that can lower blood glucose and blood lipids and have fewer side effects.

Resveratrol is a kind of polyphenol compounds, mainly derived from peanuts, grapes (red wine), knotweed, mulberries, and other plants [10]. Resveratrol is a natural antioxidant that can reduce blood viscosity. Resveratrol can also inhibit platelet coagulation and vasodilation and maintain blood flow [11]. Resveratrol has an effect on inhibiting atherosclerosis and preventing coronary heart disease, ischemic heart disease, and hyperlipidemia [12]. Because resveratrol has a variety of biological and pharmacological activities, it is widely used in food, medicine, health-care products, and cosmetics [13]. In addition, it has been found that resveratrol can control and reduce the body weight of obese rats [14]. Therefore, we speculate that resveratrol may play a role in reducing blood glucose and blood lipids in patients with GDM.

In this study, a one-time intraperitoneal injection of streptozotocin (STZ) was used to prepare a 5-day GDM rat model. The purpose of this study is to explore the regulating effect of resveratrol on blood glucose and blood lipid levels in GDM rats.

\section{Materials and Methods}

2.1. Animals. Female and male SD rats (SPF, 180-220 g) were purchased from the Hebei Experimental Animal Center (Shijiazhuang, China). All rats were reared in the animal room in a suitable breeding environment (temperature $23 \sim$ $25^{\circ} \mathrm{C}$, relative humidity $65 \sim 70 \%$ ). The photoperiod was $12 \mathrm{~h}$ : $12 \mathrm{~h}$. The animal experiment was approved by the Experimental Animal Committee of Jinan Central Hospital.

2.2. GDM Model. After the female rats were fed a high-fat diet for 8 weeks in the animal room, the estrus cycle was measured by the vaginal smear method. Female rats and male rats in pre-estrus were caged overnight at a ratio of $1: 2$. Next day, the vaginal smear was examined under a microscope, and the sperm observed was determined to be a pregnant mouse. After 5 days of pregnancy, STZ $(35 \mathrm{mg} / \mathrm{kg}$, Yi Sheng Biotechnology Co., LTD, Shanghai, China) was injected intraperitoneally to make a GDM model. After $72 \mathrm{~h}$, when the fasting blood glucose stabilized at $13.5 \mathrm{mmol} / \mathrm{L}$, the model was established successfully. 100 GDM rats were randomly divided into 5 groups: the GDM model control group (GDM-NC), resveratrol 60, 120, and $240 \mathrm{mg} / \mathrm{kg}$ treatment group, and metformin hydrochloride $(200 \mathrm{mg} / \mathrm{kg})$ positive control group. Another $20 \mathrm{SD}$ rats with 5 days gestation were taken as the normal pregnancy control group. Rats in each group were given continuous administration for 2 weeks (1 time/day). The normal pregnancy control group and GDM-NC group were given equal volume of normal saline. After 2 weeks of treatment, the rats in each group were weighed and recorded with an electronic balance.

2.3. Blood Glucose and Insulin Level. A blood glucose meter (ACCU-CHEK, Shanghai, China) was used to measure the abdominal blood glucose of each group on the 0 th, 7 th, and 14th day of treatment. The insulin level in rat plasma was measured with an ELISA kit (MILLIPORE, Beijing, China).

2.4. Detection of TC, TG, LDL-C, and HDL-C in Serum. Two weeks after the drug treatment, urethane (Beijing Chemical Reagent Company, Beijing, China) was injected intraperitoneally for anesthesia. Blood was collected from the abdominal aorta, and the upper serum was collected after centrifugation at $2000 \mathrm{rpm}$ for $5 \mathrm{~min}$. An automatic biochemical detector (Wuhan Jingcheng Weiye Medical Instruments Co., Ltd., Wuhan, China) was used to determine the serum levels of TC, TG, LDL-C, and HDL-C in each group.

2.5. Detection of Leptin, Adiponectin, Resistin, TNF- $\alpha$, and IL6 Levels in Plasma. After 2 weeks of treatment, urethane was injected intraperitoneally for anesthesia. Blood was taken from the abdominal aorta and anticoagulated with heparin. An ELISA kit (MILLIPORE, Beijing, China) was used to determine the levels of leptin, adiponectin, resistin, TNF- $\alpha$, and IL-6 in plasma.

2.6. Statistical Analysis. All experiments were repeated 3 times. SPSS 22.0 (IBM Corp.) was used for statistical analysis. All experimental data were displayed as mean \pm SD. Paired Student's $t$ test was used to analyze the parameter comparison between the two groups. $P<0.05$ was considered to indicate a statistically significant difference.

\section{Results}

3.1. The Effects of Resveratrol on Fasting Blood Glucose and Insulin Levels in GDM Rats. Compared with the normal pregnancy control group, the fasting blood glucose level of the GDM-NC group was significantly increased $(P<0.01$, Table 1). After 2 weeks of treatment with resveratrol (120 and $240 \mathrm{mg} / \mathrm{kg}$ ) and metformin hydrochloride $(200 \mathrm{mg} / \mathrm{kg})$, blood glucose levels were significantly lower than those in the GDM-NC group $(P<0.05$, Table 1$)$. Compared with the normal pregnancy control group, the insulin level of the GDM-NC group was significantly reduced $(P<0.01$, Table 2). After 2 weeks of treatment with resveratrol (120 and $240 \mathrm{mg} / \mathrm{kg})$ and metformin $(200 \mathrm{mg} / \mathrm{kg})$, insulin levels were significantly increased compared with the GDM-NC group $(P<0.05$, Table 2$)$. The abovementioned results indicate that resveratrol can increase insulin levels and lower blood glucose in GDM rats. 
TABLE 1: The effect of resveratrol on fasting blood glucose level in GDM rats.

\begin{tabular}{|c|c|c|c|c|}
\hline Group & Dosage $(\mathrm{mg} / \mathrm{kg})$ & $0^{\text {th }}$ day $(\mathrm{mmol} / \mathrm{L})$ & $7^{\text {th }}$ day $(\mathrm{mmol} / \mathrm{L})$ & $14^{\text {th }}$ day $(\mathrm{mmol} / \mathrm{L})$ \\
\hline Normal pregnancy & & $6.1 \pm 0.7^{* *}$ & $6.2 \pm 0.6^{* *}$ & $6.3 \pm 0.5^{* *}$ \\
\hline GDM-NC & & $16.3 \pm 2.2$ & $16.4 \pm 2.3$ & $16.1 \pm 2.5$ \\
\hline \multirow{3}{*}{ Resveratrol } & 60 & $16.6 \pm 2.3$ & $15.4 \pm 2.1$ & $14.9 \pm 2.1$ \\
\hline & 120 & $16.3 \pm 2.0$ & $13.5 \pm 1.8^{*}$ & $12.8 \pm 1.8^{*}$ \\
\hline & 240 & $16.2 \pm 1.8$ & $12.2 \pm 1.6^{*}$ & $9.7 \pm 1.5^{* *}$ \\
\hline Metformin hydrochloride & 200 & $16.4 \pm 2.2$ & $10.9 \pm 1.5^{*}$ & $8.8 \pm 1.3^{* *}$ \\
\hline
\end{tabular}

Compared with the GDM-NC group, $* P<0.05, * * P<0.01$.

TABLE 2: The effect of resveratrol on insulin level in GDM rats.

\begin{tabular}{|c|c|c|c|c|}
\hline Group & Dosage $(\mathrm{mg} / \mathrm{kg})$ & 0 day $(\mathrm{mmol} / \mathrm{L})$ & 7 day $(\mathrm{mmol} / \mathrm{L})$ & 14 day $(\mathrm{mmol} / \mathrm{L})$ \\
\hline Normal pregnancy & & $13.2 \pm 2.3^{* *}$ & $13.3 \pm 2.1^{* *}$ & $13.1 \pm 2.4^{* *}$ \\
\hline \multirow[t]{2}{*}{ GDM-NC } & & $5.6 \pm 1.1$ & $5.5 \pm 0.9$ & $5.6 \pm 1.0$ \\
\hline & 60 & $5.6 \pm 1.2$ & $6.8 \pm 1.4$ & $7.1 \pm 1.5$ \\
\hline \multirow[t]{2}{*}{ Resveratrol } & 120 & $5.5 \pm 0.9$ & $7.6 \pm 1.5^{*}$ & $8.5 \pm 1.3^{*}$ \\
\hline & 240 & $5.4 \pm 1.0$ & $10.3 \pm 1.6^{*}$ & $12.2 \pm 1.2^{* *}$ \\
\hline Metformin hydrochloride & 200 & $5.5 \pm 2.2$ & $7.4 \pm 1.5^{*}$ & $8.6 \pm 1.6^{*}$ \\
\hline
\end{tabular}

Compared with the GDM-NC group, ${ }^{*} P<0.05,{ }^{* *} P<0.01$.

3.2. The Effect of Resveratrol on the Weight of Rats with GDM. Compared with the normal pregnancy control group, the body weight of the GDM-NC group was increased significantly $(P<0.01$, Figure 1$)$. After 2 weeks of treatment with resveratrol (120 and $240 \mathrm{mg} / \mathrm{kg}$ ) and metformin hydrochloride $(200 \mathrm{mg} / \mathrm{kg})$, the body weight of GDM rats was significantly reduced compared with the GDM-NC group $(P<0.05$, Figure 1). These results indicate that resveratrol can reduce the body weight of GDM rats.

3.3. The Effect of Resveratrol on the Content of TC, TG, $L D L-C$, and HDL-C in the Serum of GDM Rats. Compared with the normal pregnancy control group, the serum levels of TC, TG, and LDL-C in the GDM-NC group were significantly increased, and the content of HDL-C was significantly decreased $(P<0.01$, Figures $2(\mathrm{a})-2(\mathrm{~d}))$. After 2 weeks of treatment with resveratrol (120 and $240 \mathrm{mg} / \mathrm{kg}$ ), serum levels of TC and TG were lower than those in the GDM-NC group $(P<0.05$, Figures 2(a), 2(b)). Among them, the LDL-C content of the resveratrol $(240 \mathrm{mg} / \mathrm{kg})$ treatment group was significantly reduced. Also, the HDL-C content of the resveratrol $(240 \mathrm{mg} / \mathrm{kg})$ treatment group was significantly increased $(P<0.05$, Figures $2(\mathrm{c}), 2(\mathrm{~d}))$. Compared with the metformin hydrochloride treatment group, the resveratrol $(240 \mathrm{mg} / \mathrm{kg}$ ) treatment group had a better effect on lowering blood lipids. These results indicate that resveratrol can effectively reduce blood lipid levels in GDM rats.

3.4. The Effect of Resveratrol on the Levels of Leptin, Adiponectin, Resistin, TNF- $\alpha$, and IL-6 in the Plasma of Rats with GDM. Compared with the normal pregnancy control group, the plasma levels of leptin, resistin, TNF- $\alpha$, and IL- 6 in the GDM-NC group were significantly increased, and the adiponectin level was significantly reduced $(P<0.01$, Figures 3(a)-3(e)). After 2 weeks of treatment with resveratrol $(240 \mathrm{mg} / \mathrm{kg})$, compared with the GDM-NC group, the plasma

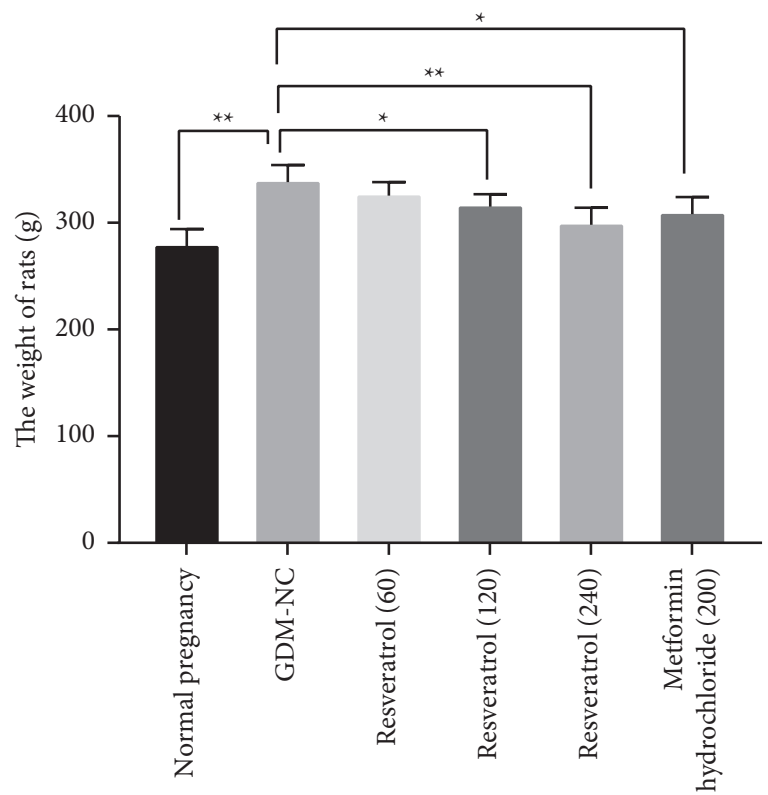

FIgURE 1: The effect of resveratrol on the weight of rats with GDM. The body weight of rats with GDM in the resveratrol $(60,120$, $240 \mathrm{mg} / \mathrm{kg}$ ) treatment group and metformin hydrochloride treatment group was compared with that in the GDM-NC group. ${ }^{*} P<0.05,{ }^{* *} P<0.01$.

levels of leptin, resistin, TNF- $\alpha$, and IL- 6 in GDM rats were significantly reduced $(P<0.05$, Figures $3(\mathrm{a}), 3(\mathrm{c})-3(\mathrm{e}))$. In addition, adiponectin was significantly increased in the $240 \mathrm{mg} / \mathrm{kg}$ resveratrol treatment group, while resistin was significantly decreased $(P<0.05$, Figure $3(\mathrm{~b}))$. Compared with the metformin hydrochloride treatment group, the plasma levels of leptin, resistin, TNF- $\alpha$, and IL- 6 in the resveratrol $(240 \mathrm{mg} / \mathrm{kg})$ treatment group were significantly increased. Also, the adiponectin level was apparently decreased (Figures 3(a)-3(e)). The abovementioned results indicate that resveratrol may reduce blood lipids in GDM rats by regulating these adipocytokines. 


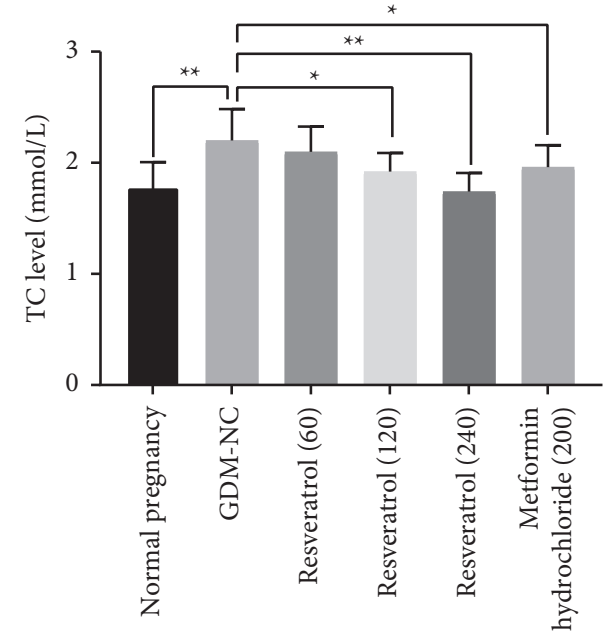

(a)

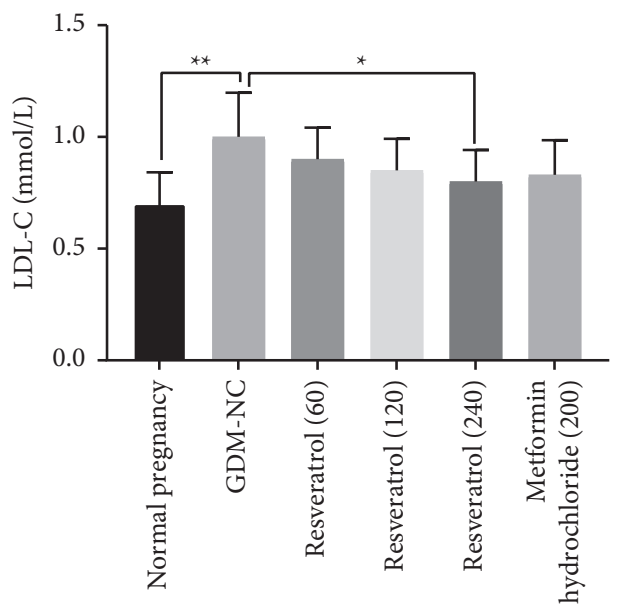

(c)

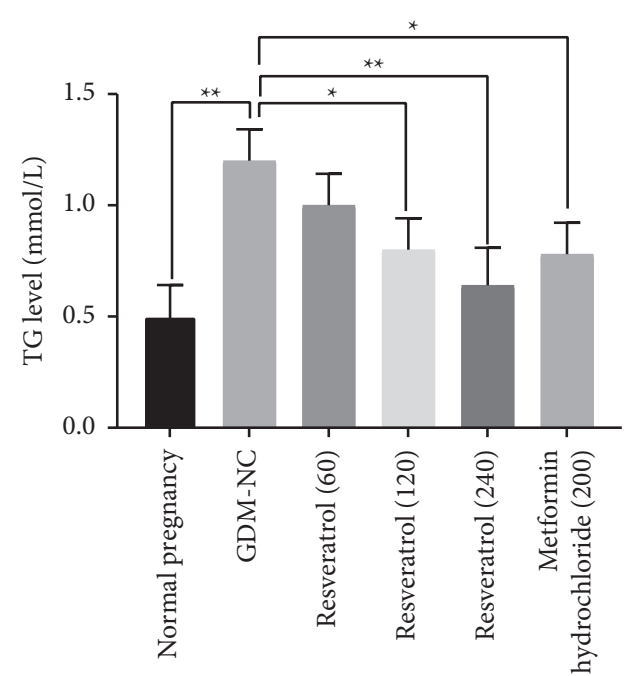

(b)

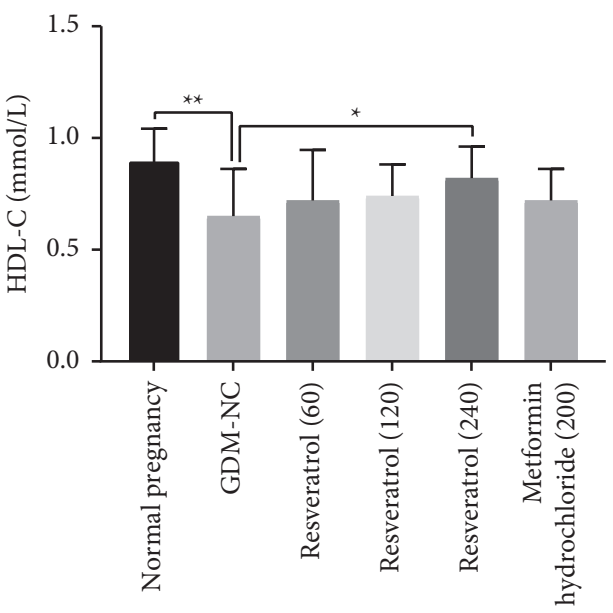

(d)

Figure 2: The effect of resveratrol on the content of TC, TG, LDL-C, and HDL-C in the serum of GDM rats. (a-d) The TC, TG, LDL-C, and HDL-C levels in of rats with GDM in the resveratrol $(60,120,240 \mathrm{mg} / \mathrm{kg})$ treatment group and metformin hydrochloride treatment group were compared with those in the GDM-NC group. $* P<0.05, * * P<0.01$.

\section{Discussion}

GDM is one of the common complications of pregnancy. Hyperglycemia during pregnancy not only endangers the health of the mother but also has an adverse effect on the fetus [15]. At present, the main clinical treatments for GDM are insulin and oral hypoglycemic drugs metformin and thiazolidinedione. However, these drugs have large side effects and easily pass through the placental barrier [16]. Therefore, the development of hypoglycemic drugs suitable for pregnant women has important clinical significance.

Resveratrol is a naturally occurring nonflavonoid polyphenol compound, which is widely present in natural plants such as grape, knotweed, cassia seed, and peanut [17]. Previous studies have reported that resveratrol has various biological effects such as anti-inflammatory, antioxidant, and antitumor $[18,19]$. In this study, we found that insulin secretion levels and fasting blood glucose levels in GDM rats were significantly increased after 2 weeks of resveratrol treatment. The levels of TC, TG, and LDL-C in the resveratrol group were significantly reduced, while the level of HDL-C was significantly increased. In addition, the weight of rats in the resveratrol group was significantly reduced. It has been shown that obesity and lipid metabolism disorders are very dangerous predisposing factors for diabetes [20]. Therefore, the hypolipidemic effect of resveratrol is of great significance to the treatment of GDM.

In addition, we also found that the levels of leptin, TNF$\alpha$, and IL- 6 in the plasma of resveratrol GDM rats were significantly reduced. Leptin has been reported to inhibit the secretion of insulin and exert a negative feedback effect between blood insulin and adipose tissue [21]. TNF- $\alpha$ and IL-6 can affect the phosphorylation of insulin-sensitive cells, block insulin signal transduction, and cause abnormal glucose metabolism [22]. This study also found that resveratrol can increase adiponectin levels and reduce resistin levels. Resistin can reduce the uptake of glucose by adipose tissue and inhibit the signal transduction pathway of 


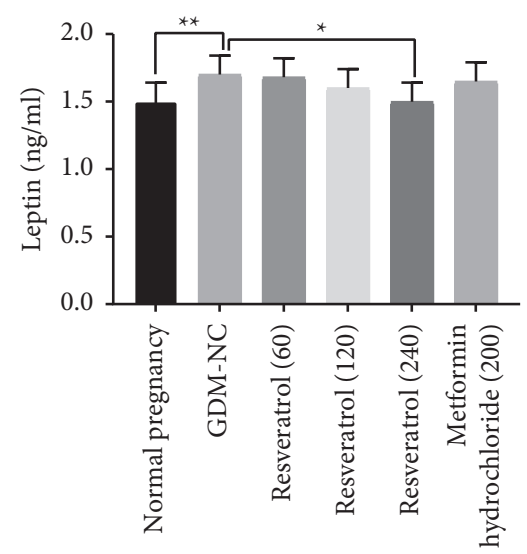

(a)

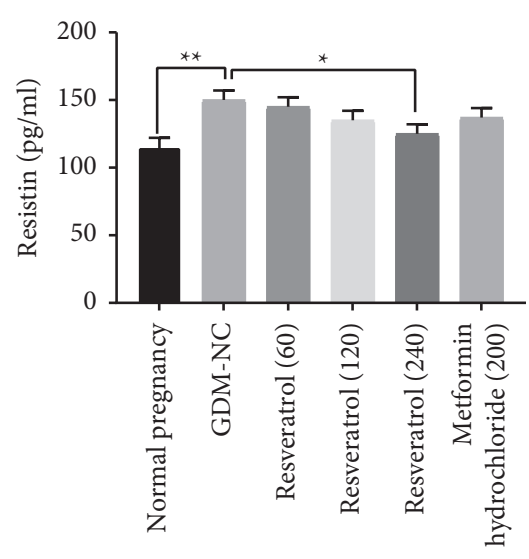

(c)

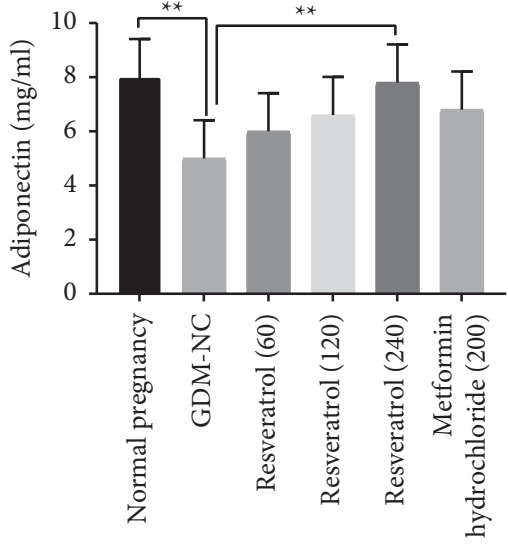

(b)

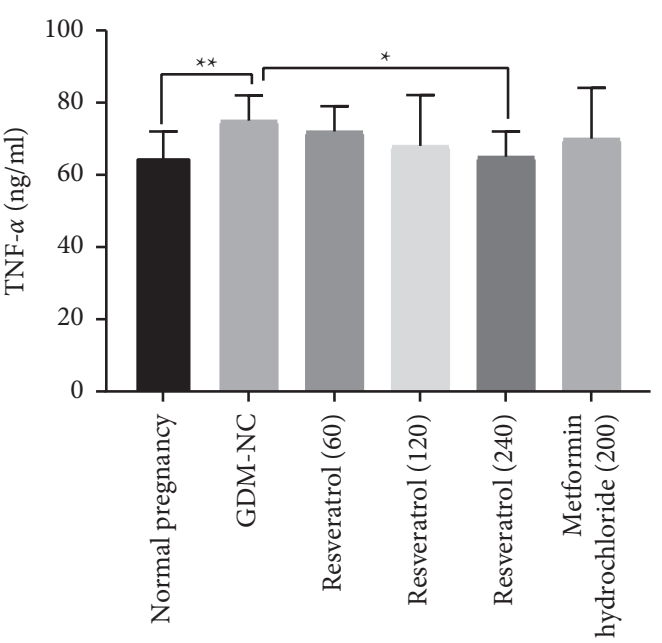

(d)

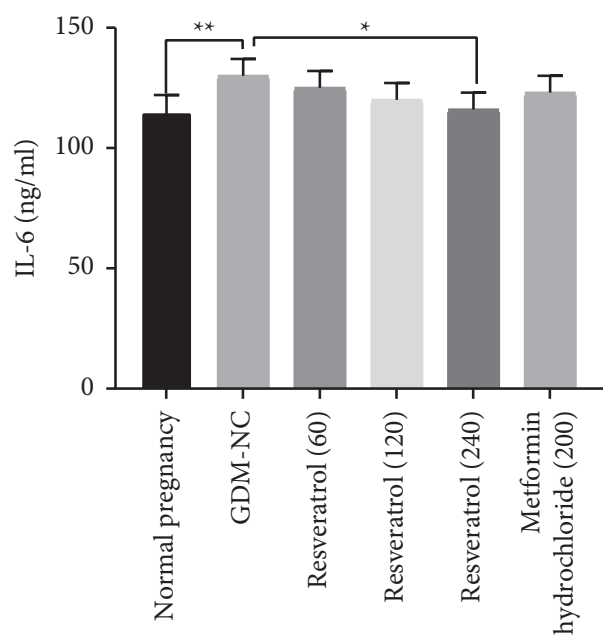

(e)

FIgURE 3: The effect of resveratrol on the levels of leptin, adiponectin, resistin, TNF- $\alpha$, and IL-6 in the plasma of rats with GDM. (a-e) The leptin, adiponectin, resistin, TNF- $\alpha$, and IL-6 levels of rats with GDM in the resveratrol $(60,120,240 \mathrm{mg} / \mathrm{kg})$ treatment group and metformin hydrochloride treatment group were compared with those in the GDM-NC group. $* P<0.05, * * P<0.01$.

insulin [23]. Adiponectin can reduce the occurrence and development of diabetes by improving insulin sensitivity [24]. These results indicate that resveratrol has a dose-dependent effect on GDM rats to increase insulin secretion, reduce blood glucose, reduce body weight, and regulate blood lipids and plasma adipokines. Moreover, resveratrol was stronger than metformin hydrochloride in improving insulin secretion and regulating blood lipids and adipokines. 
However, the specific mechanisms of resveratrol for regulating blood sugar and blood lipids are still unclear. This is also the main problem that we need to solve in the future.

\section{Conclusions}

Resveratrol has a dose-dependent effect on reducing blood glucose and blood lipids in GDM rats. Resveratrol can also treat GDM by promoting insulin secretion and regulating adipokines. However, this study has not yet elucidated the specific regulatory mechanism of resveratrol in GDM. Therefore, the regulatory mechanism of resveratrol in GDM will be explored in the future.

\section{Data Availability}

The datasets used during the present study are available from the corresponding author upon reasonable request.

\section{Conflicts of Interest}

The authors declare no conflicts of interest.

\section{References}

[1] X. C. Jiao, M. Xiao, Z. X. Gao et al., "Effects of comorbid gestational diabetes mellitus and depression on glucose metabolism during pregnancy and neonatal morphological outcome," Zhonghua Yu Fang Yi Xue Za Zhi [Chinese Journal of Preventive Medicine, vol. 54, pp. 968-973, 2020.

[2] J. Kim, Y. Piao, Y. K. Pak et al., "Umbilical cord mesenchymal stromal cells affected by gestational diabetes mellitus display premature aging and mitochondrial dysfunction," Stem Cells and Development, vol. 24, no. 5, pp. 575-586, 2015.

[3] D. J. Choi, C. H. Yoon, H. Lee et al., "The association of family history of premature cardiovascular disease or diabetes mellitus on the occurrence of gestational hypertensive disease and diabetes," PLoS One, vol. 11, no. 12, Article ID e0167528, 2016.

[4] M. Balsells, A. García-Patterson, I. Gich, and R. Corcoy, "Major congenital malformations in women with gestational diabetes mellitus: a systematic review and meta-analysis," Diabetes/Metabolism Research and Reviews, vol. 28, no. 3, pp. 252-257, 2012.

[5] X. M. Wang, Y. Gao, J. G. Eriksson et al., "Metabolic signatures in the conversion from gestational diabetes mellitus to postpartum abnormal glucose metabolism: a pilot study in Asian women," Scientific Reports, vol. 11, no. 1, Article ID 16435, 2021.

[6] S. Palomba, A. Falbo, T. Russo et al., "The risk of a persistent glucose metabolism impairment after gestational diabetes mellitus is increased in patients with polycystic ovary syndrome," Diabetes Care, vol. 35, no. 4, pp. 861-867, 2012.

[7] F. Visiedo, F. Bugatto, R. Quintero-Prado, I. Cózar-Castellano, J. L. Bartha, and G. Perdomo, "Glucose and fatty acid metabolism in placental explants from pregnancies complicated with gestational diabetes mellitus," Reproductive Sciences, vol. 22, no. 7, pp. 798-801, 2015.

[8] K. Xie, Y. Zhang, J. Wen et al., "Genetic predisposition to gestational glucose metabolism and gestational diabetes mellitus risk in a Chinese population," Journal of Diabetes, vol. 11, no. 11, pp. 869-877, 2019.
[9] M. Samimi, M. Jamilian, Z. Asemi, and A. Esmaillzadeh, "Effects of omega-3 fatty acid supplementation on insulin metabolism and lipid profiles in gestational diabetes: randomized, double-blind, placebo-controlled trial," Clinical Nutrition, vol. 34, no. 3, pp. 388-393, 2015.

[10] B. Adhikari, S. K. Dhungana, M. W. Ali, A. Adhikari, I. D. Kim, and D. H. Shin, "Resveratrol, total phenolic and flavonoid contents, and antioxidant potential of seeds and sprouts of Korean peanuts," Food Science and Biotechnology, vol. 27, no. 5, pp. 1275-1284, 2018.

[11] S. Fu, R. Lv, L. Wang, H. Hou, H. Liu, and S. Shao, "Resveratrol, an antioxidant, protects spinal cord injury in rats by suppressing MAPK pathway," Saudi Journal of Biological Sciences, vol. 25, no. 2, pp. 259-266, 2018.

[12] J. H. Ro, C. C. Liu, and M. C. Lin, "Resveratrol mitigates cerebral ischemic injury by altering levels of trace elements, toxic metal, lipid peroxidation, and antioxidant activity," Biological Trace Element Research, vol. 199, no. 10, pp. 3718-3727, 2021.

[13] M. A. Santos, F. N. Franco, C. A. Caldeira et al., "Antioxidant effect of resveratrol: change in MAPK cell signaling pathway during the aging process," Archives of Gerontology and Geriatrics, vol. 92, Article ID 104266, 2021.

[14] J. Sun, C. Zhang, M. Kim et al., "Early potential effects of resveratrol supplementation on skeletal muscle adaptation involved in exercise-induced weight loss in obese mice," BMB Reports, vol. 51, no. 4, pp. 200-205, 2018.

[15] S. G. Gray, A. N. Sweeting, T. M. McGuire, N. Cohen, G. P. Ross, and P. J. Little, "Changing environment of hyperglycemia in pregnancy: gestational diabetes and diabetes mellitus in pregnancy," Journal of Diabetes, vol. 10, no. 8, pp. 633-640, 2018.

[16] L. C. Mendoza, J. Harreiter, D. Simmons et al., "Risk factors for hyperglycemia in pregnancy in the DALI study differ by period of pregnancy and OGTT time point," European Journal of Endocrinology, vol. 179, no. 1, pp. 39-49, 2018.

[17] H. W. Wang, K. Sun, Y. X. Guan, M. H. Qiu, L. Zhang, and C. C. Dai, "Fungal endophyte phomopsis liquidambari biodegrades soil resveratrol: a potential allelochemical in peanut monocropping systems," Journal of the Science of Food and Agriculture, vol. 99, no. 13, pp. 5899-5909, 2019.

[18] M. D. Recalde, C. A. Miguel, M. V. Noya-Riobó, S. L. González, M. J. Villar, and M. F. Coronel, "Resveratrol exerts anti-oxidant and anti-inflammatory actions and prevents oxaliplatin-induced mechanical and thermal allodynia," Brain Research, vol. 1748, Article ID 147079, 2020.

[19] M. Zhu, Q. Zhang, X. Wang et al., "Metformin potentiates anti-tumor effect of resveratrol on pancreatic cancer by downregulation of VEGF-B signaling pathway," Oncotarget, vol. 7, no. 51 , pp. $84190-84200,2016$.

[20] E. Goth, "[The role of lipid metabolism disorders in obesity and diabetes]," Orvosi Hetilap, vol. 109, pp. 1977-1979, 1968.

[21] O. Ulyanova, Z. Taubaldieva, S. Tuganbekova et al., "Leptin level in patients with type 2 diabetes mellitus after fetal pancreatic stem cell transplant," Experimental and Clinical Transplantation: Official Journal of the Middle East Society for Organ Transplantation, vol. 14, pp. 45-47, 2016.

[22] S. A. Fathy, M. R. Mohamed, M. A. M. Ali, A. E. El-Helaly, and A. T. Alattar, "Influence of IL-6, IL-10, IFN-gamma and TNFalpha genetic variants on susceptibility to diabetic kidney disease in type 2 diabetes mellitus patients," Biomarkers, vol. 24, no. 1, pp. 43-55, 2019.

[23] S. E. Ghanem, M. Abdel-Samiee, M. H. Torky et al., "Role of resistin, IL-6 and NH2-terminal portion proBNP in the 
pathogenesis of cardiac disease in type 2 diabetes mellitus," BMJ Open Diabetes Research \& Care, vol. 8, 2020.

[24] G. D. Dias, F. C. Cartolano, M. C. P. Freitas et al., "Adiponectin predicts the antioxidant capacity and size of highdensity lipoprotein (HDL) in individuals with diabetes mellitus," Journal of Diabetes and its Complications, vol. 35, no. 5, Article ID 107856, 2021. 